\title{
Levantamento de Macrofungos do Reino Fungi Ocorrentes no Parque Nacional dos Campos Gerais, Paraná, Brasil
}

\author{
Natalie Alana Pedroso ${ }^{1}$, Lucila Kawana Nunes Ferreira ${ }^{1}$, Juliano Rodrigues Oliveira ${ }^{2}$ \& Lia Maris Orth Ritter Antiqueira ${ }^{3}$
}

Recebido em 13/07/2020 - Aceito em 17/11/2020

1 Universidade Tecnológica Federal do Paraná, Bolsista PIBIC Fundação Araucária. Brasil. <liamaris@gmail.com, liamaris@gmail.com>.

2 Instituto Chico Mendes de Conservação da Biodiversidade/ICMBio, Parque Nacional dos Campos Gerais. Brasil. <liamaris@gmail.com>.

3 Universidade Tecnológica Federal do Paraná, Departamento Acadêmico de Ensino, Docente do Magistério Superior da UTFPR Ponta Grossa. Brasil.<liamaris@gmail.com>.

RESUMO - O Parque Nacional dos Campos Gerais foi criado com o propósito de proteger a biodiversidade existente na região dos Campos Gerais do Paraná. A unidade de conservação federal possui mais de 21 mil hectares e abrange parte dos municípios de Ponta Grossa, Castro e Carambeí. Este estudo teve como objetivo levantar dados acerca das espécies do Reino Fungi que ocorrem na região do Parque, a fim de fornecer subsídios para elaboração do plano de manejo da unidade de conservação e contribuir com o conhecimento da biodiversidade da região, ainda pouco elucidada. Além de coleta de dados em campo foi realizada pesquisa em literatura especializada e no banco de dados speciesLink. Os resultados apontam que na região dos Campos Gerais há ocorrência de 55 espécies de fungos. Há pouca literatura disponível e poucos registros em herbários. Esses resultados revelam a necessidade urgente de estudos mais aprofundados e registro de ocorrências.

Palavras-chave: Buraco do Padre; checklist; micologia; unidade de conservação.

\section{Survey of Kingdom Fungi Macroscopic Species Occurring in the Campos Gerais National Park, Paraná, Brazil}

ABSTRACT - The Campos Gerais National Park was created to protect the existing biodiversity of the Campos Gerais region in the state of Paraná, Brazil. This federal conservation unit has more than 21 thousand hectares spread over the cities of Ponta Grossa, Castro and Carambeí. This study aimed to collect Kingdom Fungi data of species occurring in the Park so as to provide a solid basis for the conservation unit management plan as well as to better understand the park biodiversity. In addition to field data collection, research was carried out using specialized literature and the speciesLink database. Results show that there are 55 fungi species in the Campos Gerais region. Unfortunately, related literature and herbarium records are scarce. These results reveal the urgent need for more indepth studies and record of occurrences.

Keywords: Buraco do Padre; checklist; micology; conservation unity.

\section{Estudio de Macrofungos del Reino Fungi que Ocurren en el Parque Nacional Campos Gerais, Paraná, Brasil}

RESUMEN - El Parque Nacional Campos Gerais fue creado con el propósito de proteger la biodiversidad existente en la región de Campos Gerais en Paraná. La Unidad de Conservación federal tiene más de 21 mil hectáreas y cubre parte de los municipios de Ponta Grossa, Castro y Carambeí. Este estudio tuvo como objetivo recolectar datos sobre las especies del Reino Fungi que se dan en la región del Parque, con el fin de otorgar subsidios para la elaboración del Plan de Manejo de la Unidad de Conservación y contribuir con el conocimiento de la biodiversidad de la región, aún poco aclarado. Además de la recopilación de datos en el campo, se llevaron a cabo investigaciones en la literatura especializada y en la base de datos de speciesLink. Los resultados muestran que en la región de Campos Gerais hay 55 especies de hongos. Hay poca literatura disponible y pocos registros en herbario. Estos resultados revelan la urgente necesidad de estudios más profundos y registro de ocurrencias.

Palabras clave: Buraco do Padre; lista de verificación; micología; unidades de conservación. 


\section{Introdução e Embasamento do Estudo}

Os ambientes naturais dos Campos Gerais foram definidos por Maack (1948) como sendo uma zona fitogeográfica natural com campos limpos, matas de galeria, capões de floresta (floresta ombrófila mista) e fragmentos de cerrado (Savana). A vegetação é, em sua maior parte, herbácea, apresentando elementos arbustivos lenhosos. Isso se associa a regiões com floresta ombrófila mista montana, floresta ombrófila mista aluvial e remanescentes de Cerrado (Moro \& Carmo, 2007).

Embora o estado do Paraná pertença ao Domínio Mata Atlântica, possui diferentes formações vegetais (Vicente et al., 2009) e faz ecótono com o bioma Cerrado. Ecótonos são regiões de transição de diferentes ecossistemas, onde essas comunidades se encontram $e$ apresentam características e espécies particulares (Neiff, 2003; Milan \& Moro, 2016). Por tal motivo merecem especial atenção no âmbito da conservação.

A fim de proteger estes ecótonos e a biodiversidade da região dos Campos Gerais, em 2006 foi criado o Parque Nacional dos Campos Gerais (PNCG), unidade de conservação (UC) federal, atualmente gerida pelo Instituto Chico Mendes de Conservação da Biodiversidade (ICMBio), autarquia vinculada ao Ministério do Meio Ambiente.

Embora tenha sido criado em 2006, a regularização fundiária (desapropriação $e$ indenização dos proprietários de terras na área do parque) nunca foi realizada. Em paralelo, cresceu a pressão contra sua instalação, visto que a região dos Campos Gerais possui força no agronegócio e visa constante expansão da produção. Grupos políticos já solicitaram a "anulação" do decreto de criação do PNCG e mantêm constante oposição aos grupos que defendem sua manutenção $e$ regularização (pesquisadores, ambientalistas $e$ sociedade civil organizada).

O parque possui grandes atrativos turísticos, incluindo várias furnas e um rio subterrâneo. Nas proximidades da furna maior e de maior atrativo turístico, há 3 diferentes formações: mata de araucária (floresta ombrófila mista), campos limpos e remanescentes de Cerrado (Melo et al., 2005).
Alguns estudos têm se debruçado sobre o conhecimento da biodiversidade dos Campos Gerais (Antiqueira \& Moro, 2020) fornecendo elementos para subsidiar a criação do PNCG, e atualmente buscam sua regularização fundiária. Sabe-se que há muitas espécies de animais $e$ vegetais consideradas raras, endêmicas $e$ ameaçadas de extinção, vivendo nos mais variados ecossistemas. No que se refere especificamente ao Reino Fungi, não há registro de estudos nem levantamentos.

O Reino Fungi possui mais de 3.600 espécies registradas no Brasil (Forzza et al., 2010). Destas, apenas 529 são listadas como ocorrentes no estado do Paraná. Estima-se que o número de espécies conhecidas no mundo todo não represente nem a metade da biodiversidade do reino (Kirk et al., 2008).

A falta de pesquisas decorre, grande parte, da falta de iniciativas financeiras no Brasil, o que é preocupante, pois são organismos com importância ecológica, econômica, alimentícia e cosmética. Há muitas espécies ameaçadas de extinção. Taiz et al. (2017) explicam que, com a diminuição de espécies de fungos, diminui também a decomposição de matéria orgânica (processo que mantém o equilíbrio no ecossistema por meio da ciclagem de nutrientes que beneficia diversos seres vivos). Um exemplo disso são os fungos micorrízicos (que ocorrem associados a raízes de plantas, especialmente leguminosas), que possuem capacidade de enriquecer o solo, já que a planta passa a absorver melhor os nutrientes do substrato, principalmente o fósforo.

Além da decomposição de matéria orgânica, os fungos exercem funções no ambiente que permitem potencializar a economia e diminuir impactos ambientais. Por exemplo, algumas espécies consideradas entomopatogênicas, podem ser usadas no controle de insetos.

Na medicina, são de grande importância para fármacos, como é o caso do antibiótico Penicilina que é extraído da espécie Penicillium notatum ou Penicillium chrysogenum, que possui a ação de eliminar bactérias que causam infecções no corpo (Ferreira et al., 2008). São também importantes na produção de enzimas, proteínas, corantes e na área alimentícia, como os cogumelos champignon e shitake, muito usados na alimentação (Orlandelli et al., 2012; Abreu et al., 2015). 
Outro potencial de uso para as espécies do Reino Fungi é na biorremediação, especificamente na degradação de hidrocarbonetos, presentes no petróleo e gás natural. Os fungos podem limpar locais contaminados por esses combustíveis fósseis e reduzir a poluição causada por eles (Baron, 2011). Podem também contribuir na limpeza e biodegradação de efluentes (Abreu et al., 2015) e na manutenção da existência de outras espécies que os utilizam como fonte de nutrição (Macedo, 2017).

Nesse sentido, este trabalho teve como objetivo contribuir com o conhecimento da diversidade do Reino Fungi no Parque Nacional dos Campos Gerais, a fim de fornecer subsídios para elaboração do plano de manejo da unidade de conservação.

\section{Material e Métodos}

A pesquisa foi protocolada e autorizada no Sistema de Autorização e Informação em Biodiversidade (Sisbio) sob os números 71391-1 (comprovante de registro para coleta de material botânico, fúngico e microbiológico) e 71392-1 (autorização para atividades com finalidade científica).
A área de estudo (Parque Nacional dos Campos Gerais) é uma unidade de conservação federal administrada pelo ICMBio. Possui mais de 21 mil hectares e abrange parte dos municípios de Ponta Grossa, Castro e Carambeí (Figura 1), com objetivos de "preservar os ambientes naturais ali existentes com destaque para os remanescentes de Floresta Ombrófila Mista e de Campos Sulinos, realizar pesquisas científicas e desenvolver atividades de educação ambiental e turismo ecológico" (Brasil, 2006).

As coletas de exemplares macroscópicos pertencentes ao Reino Fungi foram realizadas entre outubro e dezembro de 2019 na área denominada Buraco do Padre, em Ponta Grossa, PR, nas coordenadas $25^{\circ} 10^{\prime} 18.45^{\prime \prime} S$ e $49^{\circ} 58^{\prime} 6.77^{\prime \prime} \mathrm{O}$.

Os exemplares coletados foram depositados no Herbário da Universidade Tecnológica Federal do Paraná (UTFPR), Campus Ponta Grossa. A identificação dos exemplares coletados foi realizada com base em Forzza et al. (2010).

Além das coletas, foi realizado levantamento bibliográfico e consulta ao banco de dados speciesLink (sistema distribuído de informação que integra dados primários de coleções científicas de todo o Brasil). É importante ressaltar que, embora

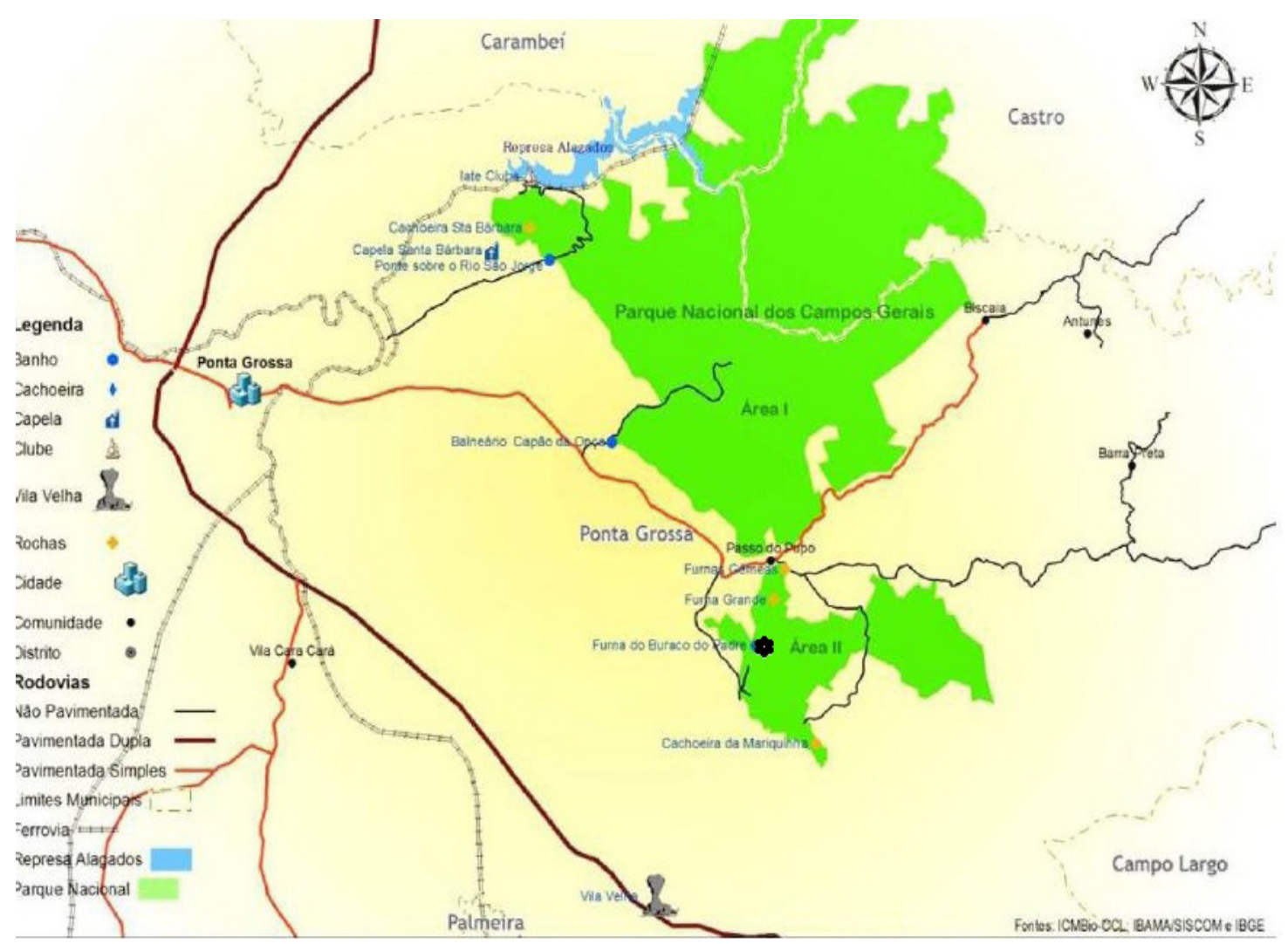

Figura 1 - Mapa de localização do Parque Nacional dos Campos Gerais. Fonte: ICMBio. 
haja outros bancos de dados e informações, não foram localizadas informações sobre a área de estudo. O speciesLink congrega o maior número de informações relativas aos Campos Gerais e por isso foi escolhido como ferramenta para essas análises. Os dados obtidos foram organizados em planilhas no Microsoft Office Excel versão 2005.

\section{Resultados e Discussão}

A pesquisa realizada no speciesLink revelou a ocorrência de 55 espécies de fungos nas áreas do PNCG. Essas espécies pertencem a dois filos e seis famílias (Quadro 1). Isso representa $11 \%$ das 529 espécies ocorrentes no Paraná, segundo dados apresentados por Forzza et al. (2010).

Quadro 1 - Espécies de fungos encontradas na região do PNCG e número de registros. Fonte: SpeciesLink.

\begin{tabular}{|c|c|c|c|}
\hline Filo & Família & Espécie & Registro \\
\hline Ascomycota & Cladoniaceae & Cladonia cartilaginea Müll.Arg & 1 \\
\hline Ascomycota & Cladoniaceae & Cladonia ceratophylla (Sw.) Spreng & 2 \\
\hline Ascomycota & Cladoniaceae & Cladonia consimilis Vainio & 1 \\
\hline Ascomycota & Cladoniaceae & Cladonia didyma (Fée) Vain. & 1 \\
\hline Ascomycota & Cladoniaceae & Cladoni adivaricata Nyl. & 1 \\
\hline Ascomycota & Cladoniaceae & Cladonia macilenta Hoffm. & 2 \\
\hline Ascomycota & Cladoniaceae & Cladonia miniata G.Meyer & 1 \\
\hline Ascomycota & Cladoniaceae & Cladonia ochrochlora Flörke & 1 \\
\hline Ascomycota & Cladoniaceae & Cladonia penicillata (Vain.) Ahti \& Marcelli & 1 \\
\hline Ascomycota & Cladoniaceae & Cladonia pityrophylla Nyl & 4 \\
\hline Ascomycota & Cladoniaceae & Cladonia pumila Ahti & 1 \\
\hline Ascomycota & Cladoniaceae & Cladonia subdelicatula Vain. ex Asah & 2 \\
\hline Ascomycota & Cladoniaceae & Cladonia verticillaris (Raddi) Fr & 1 \\
\hline Ascomycota & Parmeliaceae & Bulbothrix subcoronata (Müll.Arg.) Hale & 3 \\
\hline Ascomycota & Parmeliaceae & Canoparmelia caroliniana (Nyl.) Elix \& Hale & 6 \\
\hline Ascomycota & Parmeliaceae & Canoparmelia crozalsiana (Bouly de Les.) Elix \& Hale & 7 \\
\hline Ascomycota & Parmeliaceae & Canoparmelia nairobiensis (Stein. \& Zahlbr.) Elix \& Hale & 1 \\
\hline Ascomycota & Parmeliaceae & Canoparmelia texana (Tuck.) Elix \& Hale & 8 \\
\hline Ascomycota & Parmeliaceae & Hypotrachyna brasiliana (Nyl.) Hale. & 6 \\
\hline Ascomycota & Parmeliaceae & Hypotrachyna chlorina (Müll.Arg.) Hale & 1 \\
\hline Ascomycota & Parmeliaceae & Hypotrachyna eitenii (Hale) Hale & 1 \\
\hline Ascomycota & Parmeliaceae & Hypotrachyna endochlora (Leighton) Hale & 1 \\
\hline Ascomycota & Parmeliaceae & Hypotrachyna flavida (Zahlbr.) Hale & 6 \\
\hline Ascomycota & Parmeliaceae & Hypotrachyna intercalanda (Vain.) Hale & 1 \\
\hline Ascomycota & Parmeliaceae & Hypotrachyna livida (Tayl.) Hale & 12 \\
\hline Ascomycota & Parmeliaceae & Hypotrachyna neodissecta (Hale) Hale & 5 \\
\hline Ascomycota & Parmeliaceae & Hypotrachyna pluriformis (Nyl.) Hale & 2 \\
\hline Ascomycota & Parmeliaceae & Hypotrachyna polydactyla Krog \& Swinscow & 2 \\
\hline
\end{tabular}




\begin{tabular}{|c|c|c|c|}
\hline Ascomycota & Parmeliaceae & Hypotrachyna primitiva Hale \& López & 2 \\
\hline Ascomycota & Parmeliaceae & Hypotrachyna pustulifera (Hale) Skorepa & 4 \\
\hline Ascomycota & Parmeliaceae & Myelochroa lindmanii (Lynge) Elix \& Hale & 1 \\
\hline Ascomycota & Parmeliaceae & Parmelinopsis damaziana (Zahlbr.) Elix\&Hale & 1 \\
\hline Ascomycota & Parmeliaceae & Parmelinopsis horrescens (Taylor) Elix \& Hale & 1 \\
\hline Ascomycota & Parmeliaceae & Parmelinopsis minarum (Vain.) Elix \& Hale & 3 \\
\hline Ascomycota & Parmeliaceae & Parmelinopsis schindleri (Hale) Elix \& Hale & 10 \\
\hline Ascomycota & Parmeliaceae & Parmotrema delicatulum (Vain.) Hale & 5 \\
\hline Ascomycota & Parmeliaceae & Parmotrema dissimile Fleig & 1 \\
\hline Ascomycota & Parmeliaceae & Parmotrema mesotropum (Müll.Arg.) Hale & 5 \\
\hline Ascomycota & Parmeliaceae & Parmotrema neosubcrinitum C.H. Ribeiro \& Marcelli & 1 \\
\hline Ascomycota & Parmeliaceae & Parmotrema praeisidiosum Fleig & 1 \\
\hline Ascomycota & Parmeliaceae & Parmotrema schindleri Hale & 1 \\
\hline Ascomycota & Parmeliaceae & Parmotrema xanthinum (Müll.Arg.) Hale & 7 \\
\hline Ascomycota & Parmeliaceae & Punctelia rudecta (Ach.) Krog & 8 \\
\hline Ascomycota & Parmeliaceae & Relicina abstrusa (Vain.) Hale & 1 \\
\hline Ascomycota & Parmeliaceae & Rimelia cetrata (Ach.) Hale \& Fletcher & 17 \\
\hline Ascomycota & Parmeliaceae & Xanthoparmelia congensis(Stein.) Hale & 3 \\
\hline Ascomycota & Parmeliaceae & Xanthoparmelia hypopsila (Müll.Arg.) Hale & 2 \\
\hline Ascomycota & Parmeliaceae & Xanthoparmelia plittii (Gyelnik) Hale & 6 \\
\hline Ascomycota & Physciaceae & Heterodermia flabellata( Fée) Awasthi & 1 \\
\hline Ascomycota & Physciaceae & Heterodermia japonica (Sato) Swinscow \& Krog & 1 \\
\hline Ascomycota & Physciaceae & Heterodermia speciosa (Wulfen) Trevisan & 2 \\
\hline Ascomycota & Physciaceae & Heterodermia flabellata (Fée) Awas & 1 \\
\hline Ascomycota & Physciaceae & Physcia aipolia (Humb.) Fürn & 2 \\
\hline Basidiomycota & Polyporaceae & Trametes versicolor (L.) Lloyd & 1 \\
\hline Ascomycota & Ramalinaceae & Ramalin apusiola Müll. Arg & 4 \\
\hline
\end{tabular}

As famílias Parmeliaceae e Cladoniaceae, por meio dos gêneros Cladonia P. Browne e Bulbothrix Hale, são as que aparecem com maior frequência de registros. A espécie Rimelia cetrata é a que possui maior número de registros (17), seguida de Hypotrachyna livida, com 12 registros.

Nas atividades de campo, foram reconhecidas diversas espécies da lista obtida de literatura $e$, além dessas, foram coletadas cinco espécies. As tentativas de identificá-las se basearam em literatura (Forzza et al., 2010) e em comparação com o acervo do Herbário da Universidade Estadual de Ponta Grossa (que contempla as espécies listadas no speciesLink). Só foi possível identificar duas das espécies coletadas, sendo uma em nível de gênero (Quadro 2).

A Figura 2 apresenta uma ilustração de alguns achados fúngicos observados no PNCG e foi elaborada com finalidade didática, que é parte dos objetivos desta pesquisa científica, desenvolvida em um curso de licenciatura em Ciências Biológicas. 
Quadro 2 - Espécies coletadas no PNCG com a identificação e local de coleta/tipo de substrato.

\begin{tabular}{|c|c|c|}
\hline Filo/Família & Espécie & Local de coleta e tipo de substrato \\
\hline $\begin{array}{l}\text { Filo Ascomycota. Família Arthonia- } \\
\text { ceae }\end{array}$ & Parmotema sp & $\begin{array}{l}\text { Em árvores vivas, ocorrência comum em diversas } \\
\text { áreas de trilha nos remanescentes de Cerrado }\end{array}$ \\
\hline $\begin{array}{l}\text { Filo Basiciomycota. Família Polypo- } \\
\text { raceae }\end{array}$ & $\begin{array}{l}\text { Pycnoporus sanguineus (L.Fr.) } \\
\text { Murrill. }\end{array}$ & $\begin{array}{l}\text { Troncos de árvores mortas nos remanescentes de } \\
\text { Cerrado }\end{array}$ \\
\hline Não identificado1 & $\begin{array}{l}\text { Cogumelo pequeno de apro- } \\
\text { ximadamente } 4,5 \mathrm{~cm} \text {, branco }\end{array}$ & $\begin{array}{l}\text { Sobre rochas em locais úmidos e de pouco ilumi- } \\
\text { nação, na transição dos remanescentes de Cerrado } \\
\text { com a mata de Araucária }\end{array}$ \\
\hline Não identificado2 & $\begin{array}{l}\text { Cogumelo de aproximada- } \\
\text { mente } 20 \mathrm{~cm} \text {, cor marrom/ } \\
\text { bege }\end{array}$ & $\begin{array}{l}\text { Sobre rochas em locais úmidos e de pouca ilumina- } \\
\text { ção, na área de mata de Araucária }\end{array}$ \\
\hline Não identificado3 & $\begin{array}{l}\text { Líquen com estrutura fina } \\
\text { (semelhante a um ninho), } \\
\text { coloração verde claro }\end{array}$ & $\begin{array}{l}\text { Em árvores de diferentes espécies e sob rochas, } \\
\text { ocorrência comum na trilha de remanescentes de } \\
\text { Cerrado }\end{array}$ \\
\hline
\end{tabular}

Fonte: Os autores.

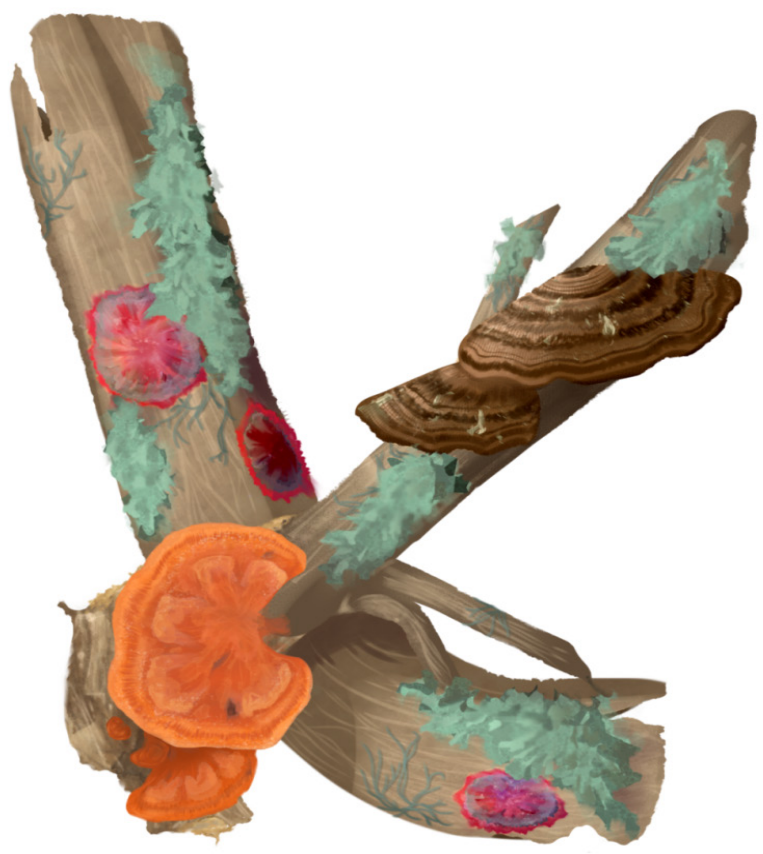

Figura 2 - Ilustração representativa de achados fúngicos no Parque Nacional dos Campos Gerais, com finalidade didática. Fonte: Pedroso, NA.

Uma das espécies identificadas é Pycnoporus sanguineus (Polyporaceae. Segundo Forzza et al. (2010), se trata de uma espécie de ampla distribuição em regiões tropicais. $\mathrm{Na}$ pesquisa no speciesLink, teve o maior número de registros, com 1572 (Figura 3). Foi a espécie que teve o maior número de cidades paranaenses nos registros (inclusive, com maior número cidades dos Campos Gerais), mostrando ser uma espécie comum e que pode viver em diferentes biomas com diferentes condições climáticas. 


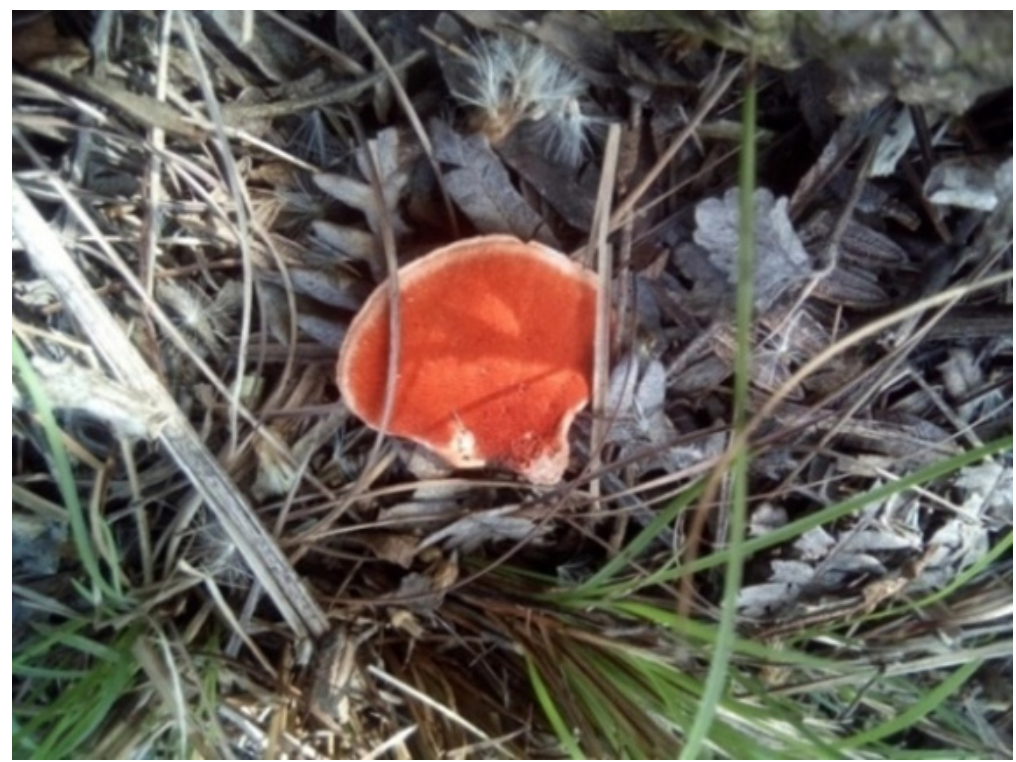

Figura 3 - Pycnoporus sanguineus (Polyporaceae) coletado no PNCG.

Pycnoporus sanguineus é um basidiomiceto que produz uma enzima pertencente a classe oxidoredutases denominada lacase. Por meio desta, degrada a lignina de diversas plantas $e$ é geralmente encontrado fixado em madeira (Hernández et al., 2016). Ao início do processo, são degradados os elementos de vasos, juntamente com o parênquima axial, e posteriormente, quando a degradação se apresenta em estágio mais avançado, degrada pelo parênquima radial $e$ nos elementos fibrosos (Ribes et al., 2018).
Além da degradação, há estudos que mostram que a enzima lacase pode contribuir no crescimento celular mais rápido, controle de doenças e até no pigmento de esporos (Leonowicz et al., 2001), evidenciando a importância da espécie. Duas espécies da família Arthoniaceae são comumente encontradas nas áreas do PNCG (Figura 4), cuja coloração varia de vermelho a branco, ou tons laranja.

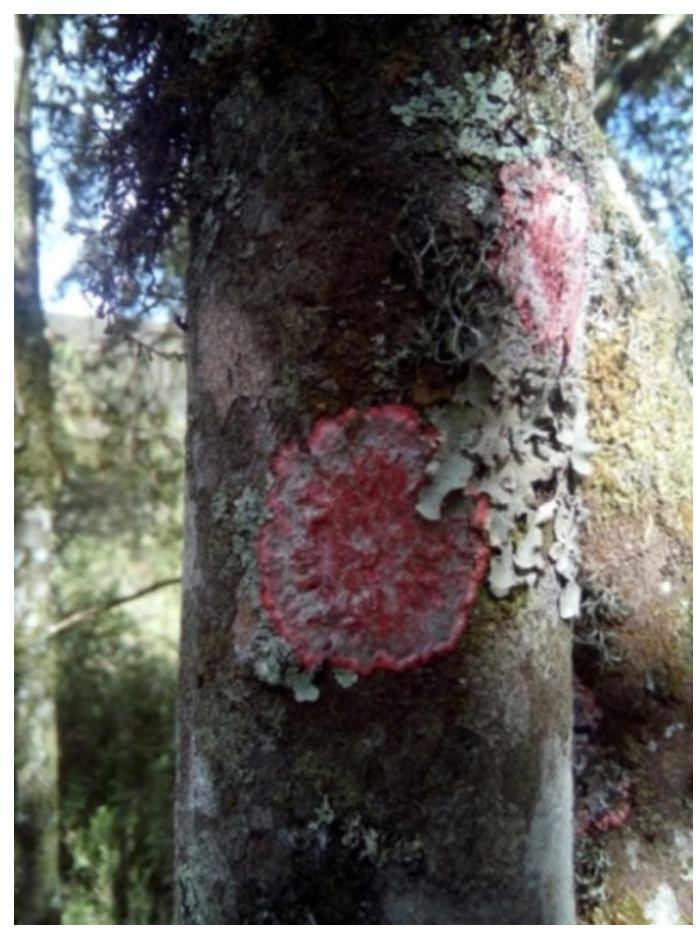

Figura 4 - Representantes da família Arthoniaceae coletados no PNCG. Fonte: Os autores. 
Essa variação se deve à sua grande sensibilidade, como muitos líquenes, a poluentes e são conhecidos e utilizados como bioindicadores. Por conta de os líquenes não possuírem estomas e nem cutículas, os aerossóis e gases acabam sendo absorvidos de forma rápida pelo talo e distribuídos pelo tecido (Riegel et al., 2019).

O fotobionte, localizado no tecido, é o primeiro a sofrer os efeitos dos poluentes, podendo ocorrer anormalidades no líquen, como o branqueamento da clorofila e o surgimento de regiões pardas nos cloroplastos. O dióxido $e$ enxofre degradam a clorofila e a transformam em feofitina (Bargagli \& Mikhailova, 2002).

A coloração, que varia de amarelo a vermelho (Figura 5), é resultado de uma hidrólise oriunda do hidróxido que forma os depsídeos e depsidonas. Alguns agrupamentos podem ligar-se ao anel aromático e mudar a coloração do líquen (Hale, 1983 apud Honda \& Vilegas, 1999).

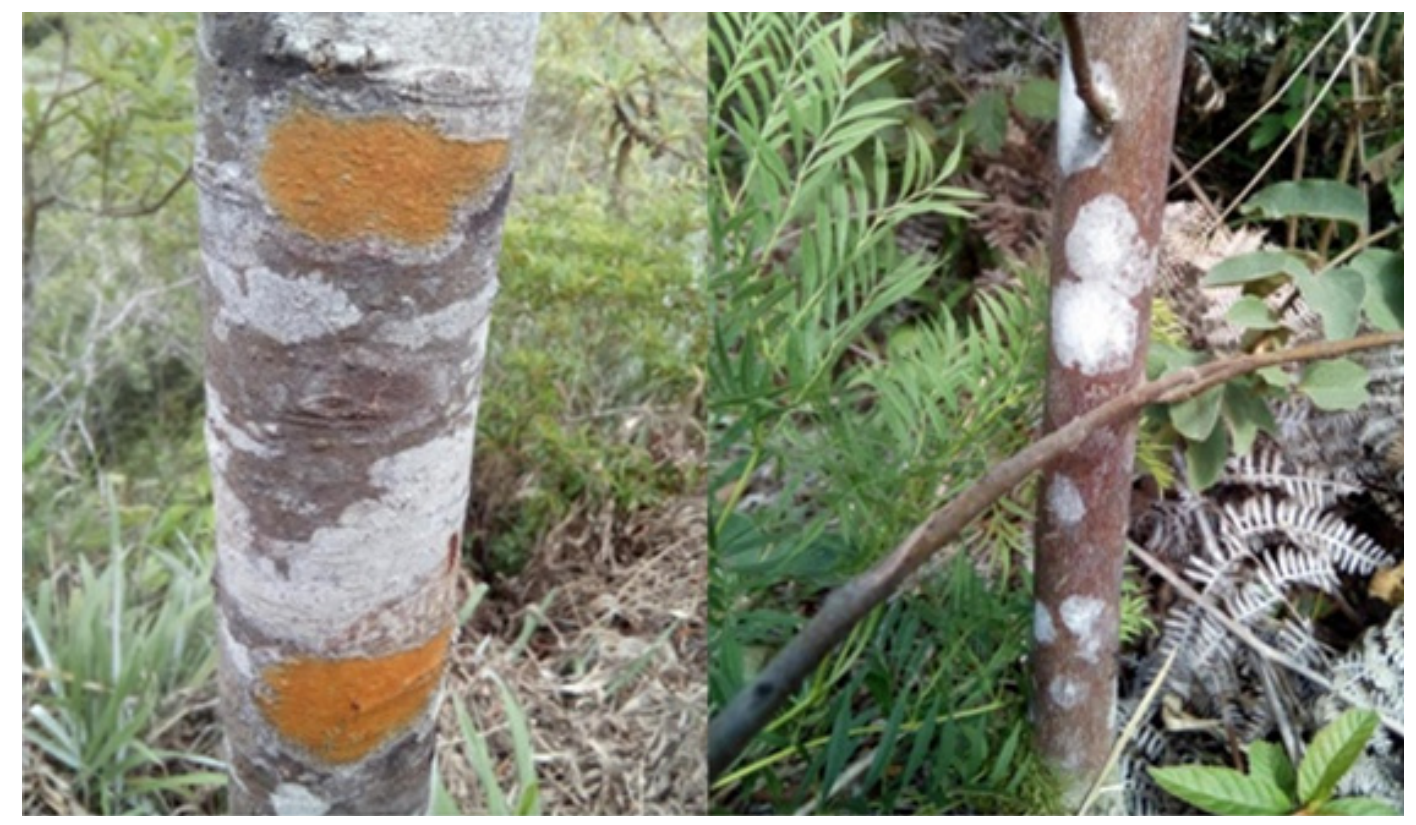

Figura 5 - Variação da cor da espécie Cryptothecia rubrocincta (Ehrenb.) G. Thor encontrada nas espécies localizadas no PNCG.

Segundo Martins et al. (2008), as espécies Heterodermia obscurata, Parmotrema tinctorum, Physcia aipolia, Teloschistes exilis e Usnea sp são bioindicadores do ar. Destas espécies citadas, uma espécie foi encontrada na região do PNCG: Physcia aipolia e dois gêneros: Heterodermia Trev e Parmotrema A. Massal também se mostraram presentes no PNCG, sendo possível observar que há mais de uma espécie considerada bioindicador na região.

A segunda espécie coletada no PNCG, foi identificada como sendo da família Arthoniaceae, gênero Parmotrema, apresentando-se como um líquen crostoso esquamuloso de cor esverdeada. Líquenes podem viver nos mais variados ambientes e locais (rochas, solo, troncos) e, muitas vezes, podem atuar como uma das primeiras formas de vida que colonizam áreas que foram expostas (Nash, 2008 apud Cavalcante, 2012). Essa associação simbiótica é presente em cerca de $20 \%$ das espécies do Reino Fungi que se tem conhecimento (Webster \& Weber, 2007 apud Cavalcante, 2012).

Segundo Barbosa (2009), diversas espécies de líquenes possuem a capacidade de absorver o nitrogênio atmosférico reduzindo o mesmo. Tal fato, contribui para o desenvolvimento de diversas espécies que utilizam do nitrogênio compartilhado pelo líquen.

Faz-se necessário investir esforços científicos no sentido de conhecer mais sobre os fungos $e$ sua biodiversidade. O desconhecimento aumenta as ameaças sob as espécies, visto que o interesse científico pode promover o manejo e a conservação destas. Na região dos Campos Gerais, poucos pesquisadores até então se debruçaram sob este 
enfoque de pesquisa, catalogando exemplares que talvez sejam raros, endêmicos ou mesmo estejam sob forte pressão antrópica.

Grande parte desse déficit de documentação vem da falta de aporte financeiro para pesquisa científica ou da dificuldade de acesso de áreas de relevante interesse científico que se encontram em propriedades particulares, visto que o próprio Parque Nacional dos Campos Gerais ainda não teve a regularização fundiária implementada.

Espera-se que estudos dessa natureza venham a contribuir também na elaboração do plano de manejo da referida unidade de conservação, haja vista a necessidade de conhecimento e gestão das áreas considerando a biodiversidade presente $e$ a conservação dos recursos naturais.

\section{Conclusão}

Os fungos dos Campos Gerais necessitam de estudos mais aprofundados. Há pouca literatura disponível e poucos registros de ocorrência $e$ exemplares depositados em herbários, como revelou a pesquisa no speciesLink. Especialmente para o Parque Nacional dos Campos Gerais, há carência absoluta de dados.

Há uma necessidade de intensificar expedições de campo, coletas para herbários e alimentação de bancos de dados de consulta pública, de forma que os registros permitam construir uma melhor visão da biodiversidade das espécies da região. É de suma importância conhecer quais as espécies de maior ocorrência, bem como a possibilidade de se identificar espécies novas, ainda não descritas pela literatura.

Este projeto de iniciação científica teve seu cronograma fortemente afetado pela pandemia de COVID-19, que cancelou as atividades presenciais impondo o isolamento social dos envolvidos, o fechamento da universidade e da unidade de conservação. Assim sendo, a repetição de coletas foi prejudicada, impondo a necessidade de reforçar os resultados com dados de literatura $e$ do banco de dados. No entanto, acredita-se que esses achados são relevantes e agregam conhecimento ao tema abordado, contribuindo com os objetivos a que se propôs a equipe.

$\mathrm{Na}$ sequência de estudos serão ser ampliados os esforços amostrais e áreas de coleta, a fim de documentar novos achados e contribuir com a implementação do Plano de Manejo do Parque Nacional dos Campos Gerais, bem como subsidiar políticas públicas voltadas para conservação de recursos naturais.

\section{Agradecimentos}

À Fundação Araucária de Apoio ao Desenvolvimento Científico e Tecnológico do Estado do Paraná, pelo apoio financeiro. Ao ICMBio pela autorização para pesquisa no PNCG (Sisbio autorização 71391-1 e 71392-1) e apoio na realização das atividades. Ao grupo de pesquisa Conservação da Natureza e Educação Ambiental (CONEA), pelo apoio nas atividades de campo e herbário.

\section{Referências}

Antiqueira LM \& Moro RS. 2020. Remanescentes de Cerrado no Parque Nacional dos Campos Gerais, PR, p. 52-58. In: Campos MA \& Azevedo DJ (orgs). Biodiversidade Brasileira: Aspectos do Estado Atual 2. Ponta Grossa: Atena. 60p.

Abreu JAS, Rovida AFS \& Pamphile JA. Fungos de Interesse: Aplicações Biotecnológicas. Universidade Estadual de Maringá - UEM. Revista UNINGÁ Review 21(1): 55-59, 2015.

Barbosa HMS. 2009. Influência do nitrogênio na produção de substâncias degradadoras do milonito por Cladonia substellata (líquen). Dissertação de Mestrado (Geografia). Universidade Federal de Pernambuco. 74 p.

Bargagli R \& Mikhailova I. 2002. Accumulation of inorganic contaminants. p. 65-84. In: Nimis PL, Scheidegger C \& Wolseley PA. (eds) Monitoring with lichens-monitoring lichens. Springer, Dordrecht. 408p.

Baron NC. 2011. Microfungos de interesse para o setor de petróleo, gás e biocombustíveis. Trabalho de Conclusão de Curso (Bacharelado em Ciências Biológicas). Universidade Estadual Paulista. 91p.

Brasil. Decreto no 4.340 de 23 de março de 2006. Brasília: Diário Oficial da União de 24 de março de 2006. < http://www.planalto.gov.br/ccivil_03/_ ato2004-2006/2006/dnn/Dnn10796.htm > Acesso em: 12/07/2020.

Cavalcante JG. 2012. Fatores associados à estrutura da comunidade de líquens corticícolas crostosos em duas áreas de caatinga no Estado de Alagoas. (Tese Mestrado em Ecologia e Conservação). Universidade Federal de Sergipe. 67p. 
Ferreira MVC, Paes VR \& Lichtenstein A. Penicilina: oitenta anos. Revista de Medicina, 87(4): 272-276, 2008.

Forzza RC et al. (orgs.). Catálogo de plantas e fungos do Brasil. vol.1. Jardim Botânico do Rio de Janeiro, Rio de Janeiro. 1: 1- 870, 2010.

Hale ME. 1983. The Biology of Lichens. 3 ed. London: Edward Arnold. 190p.

Hawksworth DL. The magnitude of fungal diversity: the 1.5 million species estimate revisited. Mycological research, 105(12): 1422-1432, 2001.

Hernández CA, Perroni Y, Pérez JAG, Rivera BG \& Alarcón E. Light-induced inhibition of laccase in Pycnoporus sanguineus. Folia microbiologica, 61(2): 137-142, 2016.

Honda NK \& Vilegas W. A química dos líquens. Química Nova, 22(1): 110-125, 1999.

Kirk PM, Cannon PF, Minter DW \& Stalpers JA. 2008. Dictionary of the Fungi. 10ed. Wallingford, UK. 784p.

Leonowicz A et al. Fungal laccase: properties and activity on lignin. Journal of Basic Microbiology: An International Journal on Biochemistry, Physiology, Genetics, Morphology, and Ecology of Microorganisms, 41: 185-227, 2001.

Maack R. 1948. Notas preliminares sobre clima, solos e vegetação do Estado do Paraná. Arquivos de Biologia e Tecnologia. vol 2. Curitiba. 225p.

Macedo EC. 2017. Principais grupos de fungos: uma interpretação com base em sua sistemática filogenética. (Tese Mestrado Profissional em Ensino de Ciências e Matemática). Instituto Federal de Educação, Ciência e Tecnologia de São Paulo. 22p.

Martins SMDA, Käffer MI \& Lemos A. Líquens como bioindicadores da qualidade do ar numa área de termoelétrica, Rio Grande do Sul, Brasil. Hoehnea, 35(3): 425-433, 2008.

Melo MS, Lopes MC \& Boska MA. 2005. Furna do Buraco do Padre, Formação Furnas/PR. p.1-11. In: Winge M, Schobbenhaus C, Berbert-Born M, Queiroz
ET, Campos DA, Souza CRG \& Fernandes ACS. (eds.), Sítios Geológicos e Paleontológicos do Brasil. < http:// sigep.cprm.gov.br/sitio110/sitio110_impresso.pdf $>$ Acesso em: 07/07/2020.

Milan E \& Moro R. O conceito biogeográfico de ecótono. Terra Plural. 10: 75-88, 2016.

Moro RS \& Carmo MRB. 2007. A vegetação campestre nos Campos Gerais, p.94-98. In: Melo MS, Moro RS \& Guimarães GB. Patrimônio Natural dos Campos Gerais do Paraná. Editora UEPG. 121p.

Nash TH. 2008. Lichen Biology. 2 ed. Cambridge, UK: Cambridge University Press. 502p

Neiff JJ. 2003. Planícies de inundação são ecótonos? p. 32-47. In: Raoul H. Ecótonos nas Interfaces dos Ecossistemas Aquáticos. Editora RIMA, São Carlos, Brazil. 350 p.

Orlandelli RC, Specian V, Felber AC \& Pamphile JA. Enzimas de interesse industrial: produção por fungos $e$ aplicações. SaBios-Revista de Saúde e Biologia, 7(3): 97-109, 2012.

Riegel J, Miranda GM \& Kemerich PDDC. Uso de líquens no monitoramento da qualidade do ar. Anais do Salão Internacional de Ensino, Pesquisa e Extensão, 10(2), 2019.

Ribes DD et al. Utilização do fungo Pycnoporus sanguineus para biopolpação de madeira. Matéria (Rio de Janeiro), 23: 1-10, 2018.

Taiz L, Zeiger E, Møller IM \& Murphy A. 2017. Fisiologia e desenvolvimento vegetal. $6^{\mathrm{a}}$ Ed. Artmed Edit. 888 p.

Valicente FH. Controle biológico de pragas, doenças e plantas invasoras. Informe Agropecuário, Belo Horizonte, 30(251): 48-55, 2009.

Vicente RF, Vanzela ALL \& Torezan JMD. Representatividade de ecossistemas no sistema de unidades de conservação no Estado do Paraná, Brasil. Natureza \& Conservação, 7(1): 50-66, 2009.

Webster J \& Weber RWS. 2007. Introduction to Fungi. $3^{\mathrm{a}}$ ed. Cambridge: Cambridge University Press. 841p.

Biodiversidade Brasileira - BioBrasil.

Edição Temática: PIBIC

n. 1,2022

http://www.icmbio.gov.br/revistaeletronica/index.php/BioBR

Biodiversidade Brasileira é uma publicação eletrônica científica do Instituto Chico Mendes de

Conservação da Biodiversidade (ICMBio) que tem como objetivo fomentar a discussão e a disseminação de experiências em conservação e manejo, com foco em unidades de conservação $e$ espécies ameaçadas.

ISSN: 2236-2886 